\title{
UCRL-JRNL-203780
}

LA WAENCE LIWEAMCAE NATIONAL LABOAATOAY

\section{Gamma-ray Imaging with Position-Sensitive HPGe Detectors}

K. Vetter, M. Burks, and L. Mihailescu,

April 19, 2004

Nuclear Instruments and Methods in Physics Research - Section 8 
This document was prepared as an account of work sponsored by an agency of the United States Government. Neither the United States Government nor the University of California nor any of their employees, makes any warranty, express or implied, or assumes any legal liability or responsibility for the accuracy, completeness, or usefulness of any information, apparatus, product, or process disclosed, or represents that its use would not infringe privately owned rights. Reference herein to any specific commercial product, process, or service by trade name, trademark, manufacturer, or otherwise, does not necessarily constitute or imply its endorsement, recommendation, or favoring by the United States Government or the University of California. The views and opinions of authors expressed herein do not necessarily state or reflect those of the United States Government or the University of California, and shall not be used for advertising or product endorsement purposes.

This work was performed under the auspices of the U.S. Department of Energy by University of California, Lawrence Livermore National Laboratory under Contract W-7405-Eng-48. 


\title{
Gamma-Ray Imaging with Position-Sensitive HPGe detectors
}

\author{
K. Vetter ${ }^{a}$, M. Burks ${ }^{\text {a }}$, L. Mihailescu ${ }^{\text {a }}$

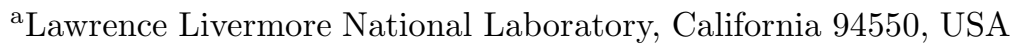

Due to advances in manufacturing large and highly segmented HPGe detectors along with the availability of fast and high precision digital electronics it is now possible to build efficient and high-resolution Compton cameras. Two-dimensionally segmented semi-conductor detectors along with pulse-shape analysis allow to obtain three-dimensional positions and energies of individual gamma-ray interactions. By employing gamma-ray tracking procedures it is possible to determine the scattering sequence in the detector and ultimately to deduce the incident direction of gamma rays without the use of a attenuating collimator. These advanced gamma-ray tracking based Compton cameras are able not only to image gamma-ray sources with higher sensitivity than collimator-based systems but can increase the sensitivity in finding gamma-ray sources over non-imaging detectors, particularly in complex radiation fields.

We have implemented a Compton camera built of a single doubule-sided strip HPGe detector with a strip pitch size of $2 \mathrm{~mm}$. A three-dimensional position resolution of $0.5 \mathrm{~mm}$ at $122 \mathrm{keV}$ by using simple pulse-shape analysis is achieved. We have implemented image reconstruction procedures for search scenarios, which are of interest for national security applications. In addition, we have developed reconstruction procedures to optimize image quality which potentially finds applications in other areas as well.

\section{Introduction}

As the title of this conference indicates and as reflected in the diversity of topics covered in this Imaging 2003 conference, imaging of photons in general and X-ray and gamma rays in particular is finding wide spread applications in research and applied sciences.

Gamma-ray imaging has emerged as an essential tool in areas such as biomedical research and nuclear medicine, high-energy astrophysics, national security and industrial non-destructive quality assessments. Well-established means for gamma-ray imaging consist of a collimator in front of a position-sensitive gamma-ray detector for uncorrelated emissions of gamma rays. A collimator is required to define the incident direction of the photon since it is not possible to focus gamma rays as in optical lens systems effectively and with large field-of-view [1]. Collimator-based systems are realized as hevimet or lead plates with parallel hole, fan-in or fan-out, single, or multiple pinholes or spatially or temporarily modulated aperture geometries [2].

However, the use of a collimator introduces a trade-off between efficiency and resolution smaller holes lead to higher resolution but lower efficiency and vice versa - and image degradation due to scattering in and penetration through the collimator. In addition, the detectors have to be shielded to prevent gamma rays entering the sensitive detector volume from the surrounding. These effects and the occurrence of Compton scattering which leads to multiple interactions degrade the performance of collimator-based gamma-ray imaging systems significantly, particularly for gamma-ray energies above $300 \mathrm{keV}$. These limitations motivate the question for ways to image gamma rays without the use of collimators. The Compton effect, which limits the sensitivity in current collimator-based systems, offers an alternative: Using the Compton scattering process and determining the energies and positions of individual interactions it is possible to deduce the incident angle without a collimator.

In the following, we will reiterate briefly the concept of Compton imaging before we discuss some of our ongoing efforts on pulse-shape analysis in two-dimensionally segmented HPGe de- 
tectors to obtain three-dimensional position information and energies of individual gamma-ray interactions. In addition, preliminary experimental results on Compton imaging will also be presented.

\section{Concept of Compton imaging}

The concept of Compton imaging was first published about thirty years ago by Todd for nuclear medicine [3] and Schoenfelder [4] for astrophysical applications. However, all attempts so far (see e.g. [5-9]) have not been able to provide the originally anticipated increase in sensitivity. Only recent developments in the manufacture of threedimensional position sensitive semi-conductor detector and advances in electronics provide the basis to realize the imaging system envisioned three decades ago $[10,11]$.

Using the Compton formula

$\cos (\vartheta)=1+\frac{511}{E_{\gamma}}-\frac{511}{E_{\gamma}^{\prime}}, E_{\gamma}^{\prime}=E_{\gamma}-E_{1}$.

it is possible to calculate the angle of the incident gamma ray by measuring the positions of the first two interactions without using a collimator. Fig. 1 illustrates the concept. A gamma ray enters the detector and interacts three times via Compton scattering until it is finally absorbed via the photo-electrical effect. The deposited energy in the first interaction and the total energy of the gamma ray determines the incident angle. Since the direction of the Compton-scattered electron is not measured it is only possible to deduce the position of the source as being on the surface of a cone. The symmetry axis of this cone is determined by the positions of the first two interactions. For image reconstruction, this cone can be projected either on a two-dimensional plane or sphere or into a three-dimensional cube. Figure 2 illustrates three such cones intersecting in one point. By analyzing the superposition of these cones the source distribution can be reconstructed. Two important components to optimize angular resolution and efficiency are obvious: Position and energy resolution, particularly of the first two interactions. Another important and ul- timately limiting factor is the intrinsic momentum of the electron the gamma-ray ray is scattering of at the first interaction. Since the momentum of the bound electron can not be measured a priori it introduces an uncertainty in the relation between energy and angle. The magnitude of the uncertainty depends on the atomic number of the scatter material, the gamma-ray energy, and the scattering.

\section{Detector configurations}

Two-dimensionally segmented HPGe detectors can be built in either coaxial or planar geometry. At LLNL, we have two planar detectors, which are built in double-sided strip (DSSD) configuration - as shown on the right of figure 3 - operational. Both DSSD detectors have a thickness of $11 \mathrm{~mm}$ and pitch sizes of $2 \mathrm{~mm}$ with $0.5 \mathrm{~mm}$ gaps in between the strips. One detector has 19 strips, the other one 39 strips on each side. Very compact and custom-made charge-sensitive preamplifiers including the FET are mounted outside the cryostat close to the signal feedthroughs. All signals are fed into a waveform digitizer-based data acquisition system manufactured by Struck Innovative Systems, which consists of 8 channel VME boards, which are read out via a VME-PCI link to a host computer. Each channel is sampled at $100 \mathrm{MHz}$ with a resolution of 12 bits. Energies and positions are determined in real time on the host computer. Energy resolution values of about $1.4 \mathrm{keV}$ at $60 \mathrm{keV}$ and $2.5 \mathrm{keV}$ at $1332 \mathrm{keV}$ are obtained; position resolution obtained with these detectors will be discussed in the next section.

In addition to the planar DSSD detectors, we have recently obtained a forty-fold segmented, closed-ended coaxial HPGe detector from ORTEC. The detector assembly including the preamplifiers can be seen on the left of figure 3 . The diameter of the n-type crystal is $5 \mathrm{~cm}$, the overall length $8.5 \mathrm{~cm}$. However, only the middle part of the coaxial region of the outside boron contact is divided in five segments longitudinally and 8 segments axially. Each segment covers 45 degrees and is $1 \mathrm{~cm}$. Due to the complexity of the electrical fields, the quasi-planar front part is not segmented and thus being left out for our imag- 
ing demonstration measurements. Another set of custom made preamplifiers with warm FETs is arranged on a circular motherboard close to the signal feedthroughs. We obtain average energy resolution values of $0.94 \mathrm{keV}$ at $60 \mathrm{keV}$ and $1.95 \mathrm{keV}$ at $1332 \mathrm{keV}$. To be able to determine positions and energies of individual interactions based on pulseshape analysis, we performed measurements to map out signals from single interactions throughout the detector, similar to [12]. Based on these measurements we calculated sets of signals which are used to fit measured signals and to decompose them into individual gamma-ray interactions [13]. We have just obtained the first Compton images from this detector, however, below we will focus on results obtained with the planar DSSD systems. Results from our coaxial detector will be reported in a forthcoming paper [14].

\section{Position sensitivity}

Pulse-shape analysis is required in planar DSSD as well as in two-dimensionally segmented coaxial HPGe detectors to determine the depth or the radial position, respectively. However, pulseshape analysis can also be applied to improve the complimentary position resolution beyond the segmentation size. How this is done in coaxial detectors is extensively discussed in [12]. Here, we will briefly discuss how the position resolution can be improved beyond the strip dimension in DSSD systems. Details can be found in a forthcoming publication [15]. Figure 4 shows signals obtain from a single gamma-ray interaction. Positive as well as negative normalized net charge signals from the opposing strips are shown as well as transient charge signals from both adjacent neighbors. As demonstrated in $[16,17,10]$ the depth can be determined simply by the rise time difference between both net charge signals. Either a constant fraction method can be applied or just the time to reach $50 \%$ of the amplitude can be used as rise time reference. To improve the resolution beyond the strip size (in $\mathrm{X}$ and $\mathrm{Y}$ ) the difference between the maximum amplitudes (A) of transient charge signals in adjacent strips can be used:

$X \propto \frac{\left(A_{R}-A_{L}\right)}{A_{R}+A_{L}}, Y \propto \frac{\left(A_{T}-A_{B}\right)}{A_{T}+A_{B}}$

Here, $\mathrm{X}$ refers to the horizontal dimension, $\mathrm{A}_{R}$ and $\mathrm{A}_{L}$ are the amplitudes of the right and left neighbor, respectively. Y refers to the vertical dimension, $\mathrm{A}_{T}$ and $\mathrm{A}_{B}$ are the amplitudes of the top and bottom neighbor, respectively. Results with this approach are shown in figure 5. Here, data from three different locations of a multi-layer collimation system are shown. A ${ }^{57} \mathrm{Co}$ source was collimated to $0.5 \mathrm{~mm}$ in diameter and netcharge as well as transient-charge signals of anode and cathode were analyzed according to the procedures discussed above. On the left, measured X (AC) positions are plotted as a function of measured depths values. Nonlinearities which can be seen between 1 and $4 \mathrm{~mm}$ in depth reflect the weighted potentials of the individual strips. While the nonlinearities can be corrected, the marked region with less number of events is characterized by a reduced position sensitivity. This is due to the cancellation of holes and electrons, which results in small transient signals. The figure on the right shows the $\mathrm{X}-\mathrm{Y}$ positions measured at the three different collimator locations. We obtain an overall position resolution of $0.5 \mathrm{~mm}$ at an energy of $122 \mathrm{keV}$ in all three dimensions.

\section{Experimental results on Compton imag- ing}

Using the positions we obtained in the previous section and combining them with the energies which have been measured on the strips we can identify individual gamma-ray interactions with their corresponding positions and energies and extract the scattering sequence by employing gamma-ray tracking procedures, e.g. as demonstrated in [18]. Compton images can be obtained by projecting the cones, that are constructed by using the positions of the two first interactions and the energy of the first interaction and the total gamma-ray energy. By adding many event cones to the projection an image can be generated. Applying list-mode maximum likelihood minimization procedures [19] we can improve the 
image quality as indicated in figure 5. Only after employing image reconstruction procedures it is possible to obtain the instrument limited angular resolution, which enables to recover the two ${ }^{22} \mathrm{Na}$ sources which were separated by 10 degrees. In addition to these imaging measurements we have performed measurements to demonstrate the increased sensitivity of our DSSD HPGe detector when operated in Compton imaging mode over the operation in non-imaging mode.

\section{Conclusions}

We have assembled two-dimensionally segmented coaxial and planar double-sided strip HPGe detectors as Compton imaging devices. While Compton imaging with the coaxial detector is still in a premature state we have two DSSD HPGe detectors operational as Compton imagers. Pulse-shape analysis algorithms have been developed and implemented which provide a position resolution of $0.5 \mathrm{~mm}$ at $122 \mathrm{keV}$ in all three dimensions. Gamma-ray tracking as well as image reconstruction procedures have been developed and implemented as well. We are now able to demonstrate the increased sensitivity of Compton imaging over non-imaging detectors.

\section{Acknowledgements}

We would like to express our gratitude to Ethan Hull who manufactured the DSSD HPGe detectors, Klaus Ziock who made the detectors available, Dean Beckedahl and Jerry Blair who were essential in the design of the coaxial HPGe detector, and Greg Schmid who did significant amount of ground work for our emerging Compton imaging program.

The work was performed under the auspices of the U.S. Department of Energy by University of California Lawrence Livermore National Laboratory under contract No. W-7405-Eng-48.

\section{REFERENCES}

1. Pivovaroff et al., J. Nucl. Med. 43,5 (2002) 231

2. Physics in Nuclear Medicine, 2nd ed., J.A.
Sorenson and M. Phelps; W. B. Saunders Company: Philadelphia, PA 1987

3. R.W. Todd, J.M. Nightingale, and D.B. Everett, Nature 251 (1974) 132

4. V. Schoenfelder et al., Nucl. Instr. and Meth. 107 (1973) 385

5. M. Singh, Medical Physics 10(4) (1984) 421

6. G.W. Philips, Nucl. Instr. and Meth. B99 (1995) 674

7. B.F Phlips, et al., IEEE Trans. Nucl. Sci. 43(3) (1996) 1472

8. J.W. LeBlanc, et al., IEEE Trans. Nucl. Sci. 45 (1998) 943

9. G.J. Schmid et al., Nucl. Instr. and Meth. A430 (1999) 69

10. E.A. Wulf, et al., IEEE Trans. Nucl. Sci. NS49 (2002) 1876

11. R.A. Kroeger, et al., IEEE Trans. Nucl. Sci. NS-49 (2002) 1887

12. K. Vetter, et al., Nucl. Instr. and Meth. A 452 (2000) 223

13. K. Vetter, Nucl. Phys. News, 12,2 (2002) 15

14. T. Niedermayr, et al., to be published in Nucl. Instr. and Meth.

15. M. Burks, et al., to be published in Nucl. Instr. and Meth.

16. M. Momayezi, W.K. Warburton, R. Kroeger, SPIE 3768 (1999) 530

17. M. Amman, P.N. Luke, Nucl. Instr. and Meth. A 452 (2000) 155

18. G.J. Schmid, et al., Nucl. Instr. and Meth. A430 (1999) 69

19. L. Mihailescu, et al., to be published in Nucl. Instr. and Meth. 


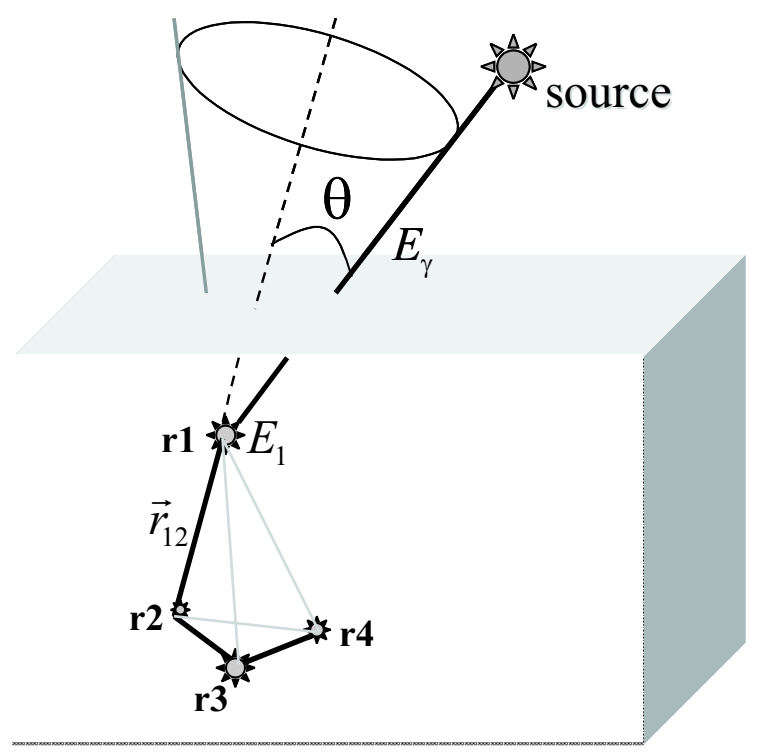

Figure 1. Fig. 1: Concept of collimator-less Compton imaging.

\section{Figures}

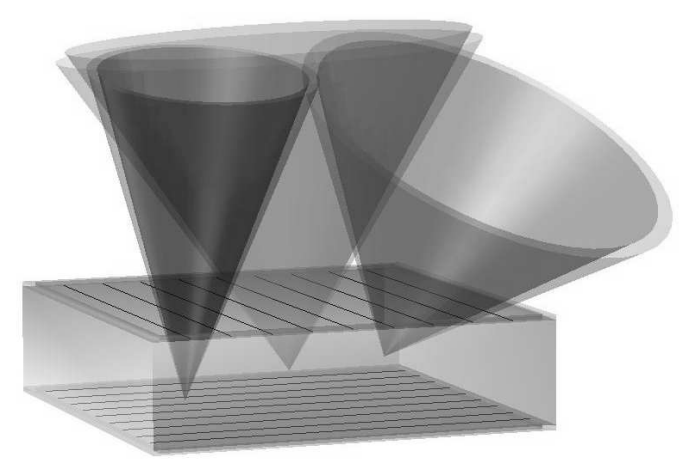

Figure 2. Fig. 2: Three back projected cones which intersect in one point which is the source location.
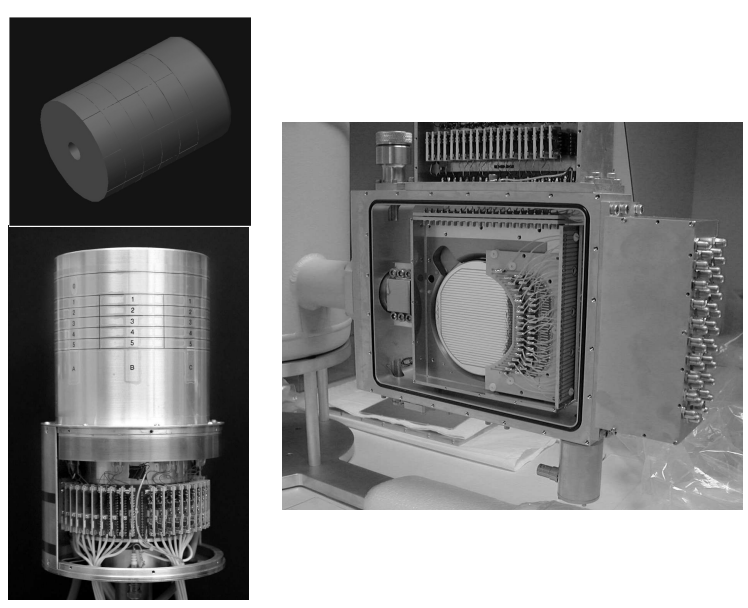

Figure 3. Fig. 3: Two possible implementations of two-dimensionally segmented HPGe detectors: a forty-fold segmented, closed-ended coaxial HPGe detector on the left and a 2x39fold segmented double-sided strip planar HPGe detector on the right. 


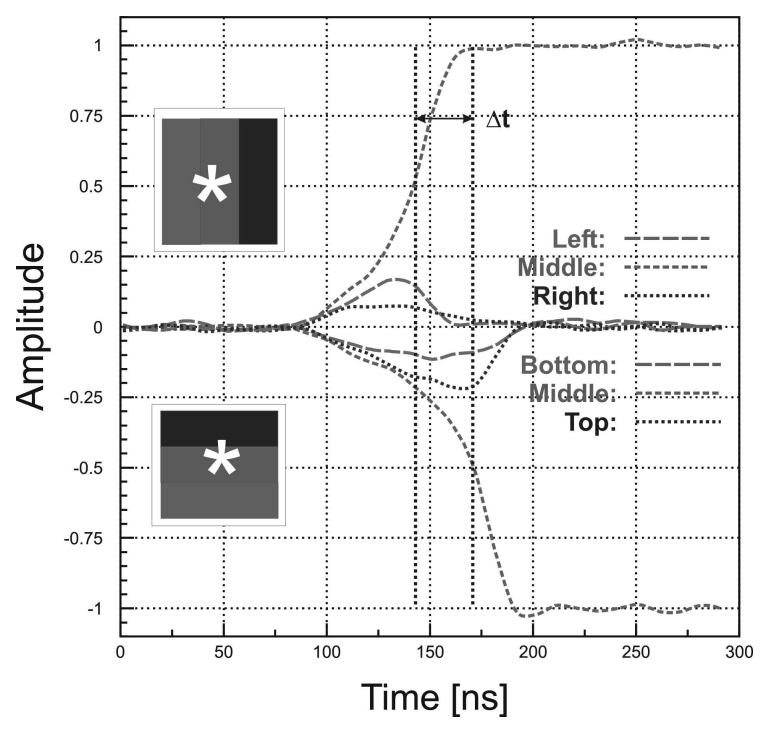

Figure 4. Fig. 4:Normalized signals measured from a single interaction in a DSSD HPGe detector. Shown are net-charge signals from opposing strips with opposing polarity and transientcharge signals from adjacent strips. The positive polarity signals determine the horizontal position, the negative polarity signals the vertical position.
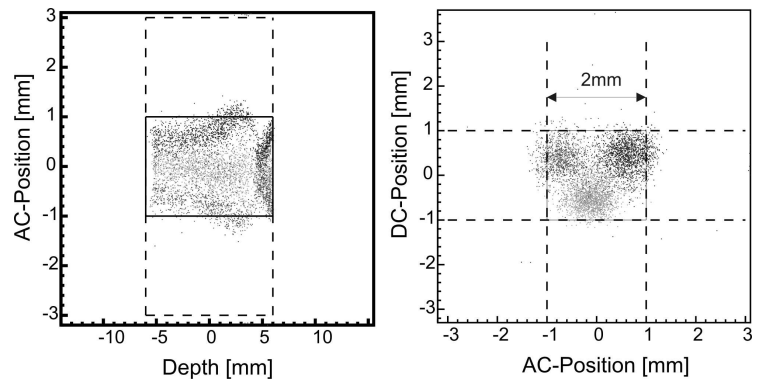

Figure 5. Fig. 5: Demonstration of the achieved three-dimensional position sensitivity in a DSSD HPGe detector. Shown are two-dimensional projections of interaction positions at $122 \mathrm{keV}$, which have been extracted by simple pulse-shape analysis. The nonlinearities in depth, which are visible on the left plot reflect the weighted potentials for this segment configuration. The white area indicates the volume in which electrons and holes are compensating each other which results in small transient signals and therefore reduced position sensitivity.
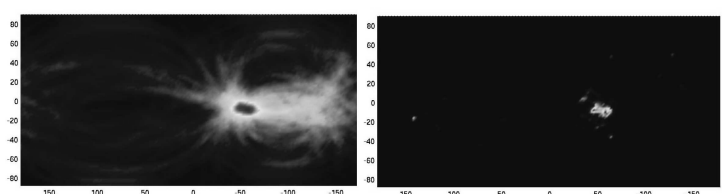

Figure 6. Fig. 6:Compton images of two ${ }^{22} \mathrm{Na}$ sources which were separated by 10 degrees. The images were obtained with the DSSD HPGe detector and employing a list-mode maximum likelihood Image reconstruction. 